\title{
Packing densities of patterns
}

\author{
Reid W. Barton \\ Department of Mathematics \\ Massachusetts Institute of Technology \\ Cambridge, MA 02139 \\ rwbarton@mit.edu
}

Submitted: Aug 8, 2004; Accepted: Nov 5, 2004; Published: Nov 12, 2004

Mathematics Subject Classifications: Primary 05A15; Secondary 05A16

\begin{abstract}
The packing density of a permutation $\pi$ of length $n$ is the maximum proportion of subsequences of length $n$ which are order-isomorphic to $\pi$ in arbitrarily long permutations $\sigma$. For the generalization to patterns $\pi$ which may have repeated letters, two notions of packing density have been defined. In this paper, we show that these two definitions are equivalent, and we compute the packing density for new classes of patterns.
\end{abstract}

\section{Definition of packing density}

A pattern or word is a string of letters from a totally ordered alphabet $\Sigma$. Two patterns $\pi_{1}$ and $\pi_{2}$ are said to be order-isomorphic (or simply isomorphic) if they have the same length and any two symbols of $\pi_{1}$ have the same order relation $(<,>$, or $=)$ as the symbols in the corresponding positions in $\pi_{2}$. An occurrence of a pattern $\pi$ in a word $\sigma$ is a subsequence of symbols of $\sigma$ (not necessarily consecutive) which form a pattern order-isomorphic to $\pi$.

We use the notation $[k]^{n}$ for the set of all $n$-letter words on the standard $k$-letter alphabet $[k]:=\{1,2, \ldots, k\}$. The terms "pattern" and "word" are interchangeable, but we will generally use them as in the last sentence of the previous paragraph, that is, we are counting occurrences of patterns in words. Note that since $\sigma$ can contain repeated symbols, multiple occurrences of $\pi$ in $\sigma$ can be equal as sequences of letters in $\Sigma$, but should be counted as distinct occurrences. For example, the word $\sigma=122$ contains the pattern $\pi=12$ twice, not once.

Definition 1 Let $\Pi$ be a collection of patterns of length $m$, no two of which are isomorphic. For a word $\sigma \in[k]^{n}$, we define $\nu(\Pi, \sigma)$ to be the total number of occurrences of patterns $\pi \in \Pi$ in $\sigma$. The density of $\Pi$ in $\sigma$ is then $d(\Pi, \sigma):=\nu(\Pi, \sigma) /\left(\begin{array}{c}n \\ m\end{array}\right)$. 
We require that patterns in $\Pi$ be non-isomorphic to ensure that any subsequence of $\sigma$ of length $m$ matches at most one pattern in $\Pi$. It is then easy to see that $d(\Pi, \sigma)$ is the probability that a randomly selected subsequence of $\sigma$ of length $m$ will be orderisomorphic to some $\pi \in \Pi$. In particular, $0 \leq d(\Pi, \sigma) \leq 1$. In the remainder of this paper, we will always implicitly assume that the patterns in $\Pi$ are non-isomorphic.

Definition 2 For $\Pi$ a collection of patterns of length $m$, define

$$
\delta(\Pi, k, n):=\max \left\{d(\Pi, \sigma) \mid \sigma \in[k]^{n}\right\} .
$$

Our general goal in studying packing density problems is to understand the behavior of this function $\delta(\Pi, k, n)$. We call a pattern $\sigma \in[k]^{n}$ such that $d(\Pi, \sigma)=\delta(\Pi, k, n)$ a $\Pi$ maximizer among all patterns in $[k]^{n}$. For specific sets of patterns $\Pi$, one can often show that there exists a $\Pi$-maximizer $\sigma$ of a form similar to the form of the patterns $\pi \in \Pi$, and then compute $\delta(\Pi, k, n)$ by maximizing $d(\Pi, \sigma)$ over all such words of this form. We now investigate the behavior of $\delta(\Pi, k, n)$ for general $\Pi$, specifically how it varies with $k$ and $n$. The following result is standard in the literature, but we reproduce it here for completeness.

Proposition 1 Let $\Pi$ be a collection of patterns of length $m$. Then

(a) $\delta(\Pi, k+1, n) \geq \delta(\Pi, k, n)$, with equality for $k \geq n$;

(b) $\delta(\Pi, k, n+1) \leq \delta(\Pi, k, n)$ for $n \geq m$.

Proof. Part (a) is clear, since every $\sigma \in[k]^{n}$ is also in $[k+1]^{n}$, and when $k \geq n$, every $\sigma \in[k+1]^{n}$ contains at most $n$ distinct letters and is therefore order-isomorphic to an element of $[k]^{n}$.

For part (b), assume $n \geq m$, and let $\sigma \in[k]^{n+1}$ be a $\Pi$-maximizing word, i.e., one for which $d(\Pi, \sigma)=\delta(\Pi, k, n+1)$. For $1 \leq i \leq n$, let $\sigma_{i} \in[k]^{n}$ be the word obtained by omitting the $i$ th symbol of $\sigma$. Since $n+1>m$, we can select a random subsequence of $\sigma$ of length $m$ by first throwing out one of the $n+1$ symbols of $\sigma$ at random and then randomly selecting a subsequence of length $m$ from the resulting word. Using the probabilistic interpretation of $d(\Pi, \sigma)$, we conclude that $d(\Pi, \sigma)$ equals the average of the $d\left(\Pi, \sigma_{i}\right)$. In particular, for some $i$ we have $d\left(\Pi, \sigma_{i}\right) \geq d(\Pi, \sigma)=\delta(\Pi, k, n+1)$, so $\delta(\Pi, k, n) \geq \delta(\Pi, k, n+1)$, as desired.

These inequalities allow us to define various limits of $\delta(\Pi, k, n)$ as $k$ and $n$ increase. First, note that for $n \geq m$, we have $\delta(\Pi, n, n)=\delta(\Pi, n+1, n) \geq \delta(\Pi, n+1, n+1)$, so the sequence $\delta(\Pi, n, n)$ is nonincreasing and bounded, hence converges. Also, for fixed $k$, the sequence $\delta(\Pi, k, n)$ is nonincreasing, and the limit is an increasing function of $k$. Therefore the following limits are all defined.

Definition 3 For $\Pi$ a collection of patterns of length $m$, define

$$
\begin{aligned}
\delta(\Pi) & =\lim _{n \rightarrow \infty} \delta(\Pi, n, n), \\
\delta(\Pi, k) & =\lim _{n \rightarrow \infty} \delta(\Pi, k, n), \\
\delta^{\prime}(\Pi) & =\lim _{k \rightarrow \infty} \delta(\Pi, k) .
\end{aligned}
$$


Note that $\delta(\Pi)=\lim _{n \rightarrow \infty} \lim _{k \rightarrow \infty} \delta(\Pi, k, n)$ and $\delta^{\prime}(\Pi)=\lim _{k \rightarrow \infty} \lim _{n \rightarrow \infty} \delta(\Pi, k, n)$; the only difference is in the order of limiting operations.

Example 1 [2, Example 1.3] Let $\Pi$ be the collection containing the single pattern $\pi=$ $12 \cdots m$. Note that for any $n \geq m$, the word $\sigma=12 \cdots n$ gives $d(\Pi, \sigma)=1$, so $\delta(\Pi, n, n)=$ 1 and therefore $\delta(\Pi)=1$.

To compute $\delta^{\prime}(\Pi)$, let $k \geq m$ and $r \geq 1$, and consider the word $\sigma=1^{r} 2^{r} \cdots k^{r}$; this notation means that each of the symbols $1,2, \ldots, k$ is repeated $r$ times. We obtain an occurrence of $\pi$ in $\sigma$ by first picking $m$ of the letters $1,2, \ldots, k$, and then for each letter picking one of its $r$ occurrences; thus $\nu(\Pi, \sigma)=\left(\begin{array}{c}k \\ m\end{array}\right) r^{m}$ and

$$
\delta(\Pi, k, r k) \geq d(\Pi, \sigma)=\frac{\left(\begin{array}{c}
k \\
m
\end{array}\right) r^{m}}{\left(\begin{array}{c}
r k \\
m
\end{array}\right)} .
$$

Therefore

$$
\delta(\Pi, k)=\lim _{r \rightarrow \infty} \delta(\Pi, k, r k) \geq \lim _{r \rightarrow \infty} \frac{\left(\begin{array}{c}
k \\
m
\end{array}\right) r^{m}}{\left(\begin{array}{c}
r k \\
m
\end{array}\right)}=\frac{\left(\begin{array}{c}
k \\
m
\end{array}\right)}{k^{m} / m !}
$$

which approaches 1 as $k \rightarrow \infty$. Hence $\delta^{\prime}(\Pi)=1$.

Observe that for this $\Pi, \delta(\Pi)=\delta^{\prime}(\Pi)$. In [2], the authors extend this result to sets of patterns $\Pi$ of a special type and raise the question of whether equality holds in general. Theorem 3 answers this question in the affirmative, using the technique of repeating letters as in the example above.

Lemma 2 Let $\Pi$ be a collection of patterns of length $m$ and let $\sigma \in[n]^{n}$ be a $\Pi$-maximizer. Then

$$
\left(\begin{array}{c}
n \\
m
\end{array}\right) \delta(\Pi) \leq \nu(\Pi, \sigma) \leq \frac{n^{m}}{m !} \delta^{\prime}(\Pi)
$$

Proof. Since $\delta(\Pi, n, n) \geq \delta(\Pi)$ there exists $\sigma \in[n]^{n}$ with $d(\Pi, \sigma) \geq \delta(\Pi)$, so $\nu(\Pi, \sigma)=$ $\left(\begin{array}{c}n \\ m\end{array}\right) d(\Pi, \sigma) \geq\left(\begin{array}{c}n \\ m\end{array}\right) \delta(\Pi)$, proving the left-hand inequality. For the other inequality, let $\sigma \in[n]^{n}$ be any word; we must show that $\nu(\Pi, \sigma) \leq \frac{n^{m}}{m !} \delta^{\prime}(\Pi)$. For $r \geq 1$, define the word $\sigma_{r} \in[n]^{r n}$ by repeating each letter of $\sigma r$ times. Every occurrence of a pattern $\pi \in \Pi$ in $\sigma$ gives rise to $r^{m}$ occurrences of $\pi$ in $\sigma_{r}$, so $\nu\left(\Pi, \sigma_{r}\right) \geq r^{m} \nu(\Pi, \sigma)$. Therefore

$$
\delta(\Pi, n)=\lim _{r \rightarrow \infty} \delta(\Pi, n, r n) \geq \lim _{r \rightarrow \infty} d\left(\Pi, \sigma_{r}\right) \geq \lim _{r \rightarrow \infty} \frac{r^{m} \nu(\Pi, \sigma)}{\left(\begin{array}{c}
r n \\
m
\end{array}\right)}=\frac{m !}{n^{m}} \nu(\Pi, \sigma) .
$$

Since $\delta^{\prime}(\Pi) \geq \delta(\Pi, n)$, we obtain the right-hand inequality.

Theorem 3 Let $\Pi$ be a collection of patterns of length $m$. Then $\delta(\Pi)=\delta^{\prime}(\Pi)$.

Proof. First note that $\delta(\Pi, k, k) \geq \delta(\Pi, k)$ for $k \geq m$, so

$$
\delta(\Pi)=\lim _{k \rightarrow \infty} \delta(\Pi, k, k) \geq \lim _{k \rightarrow \infty} \delta(\Pi, k)=\delta^{\prime}(\Pi) .
$$

On the other hand, letting $n \rightarrow \infty$ in the lemma shows that $\delta(\Pi) \leq \delta^{\prime}(\Pi)$.

Definition 4 The packing density of $\Pi$ is the common value $\delta(\Pi)=\delta^{\prime}(\Pi)$. 


\section{Layered permutations and clumpy patterns}

In the study of packing densities of permutations, it has been found that the easiest permutations to analyze are the so-called layered permutations.

Definition 5 A layered permutation is an increasing sequence of decreasing segments, in other words, a permutation which can be written $\pi=\pi_{1} \pi_{2} \cdots \pi_{l}$ where $\pi_{1}<\pi_{2}<\cdots<\pi_{l}$ and each $\pi_{i}$ is a decreasing sequence. Here $\pi_{i}<\pi_{i+1}$ means that every letter in $\pi_{i}$ is less than every letter in $\pi_{i+1}$. The segments $\pi_{i}$ are called the layers of $\pi$.

A typical example of a layered permutation is $\pi=321465$; this permutation has three layers, with sizes 3,1 , and 2 . Note that a layered permutation is uniquely determined by its sequence of layer sizes. We will use the notation $\left[k_{1}, \ldots, k_{l}\right]$ for the layered permutation with layer sizes $k_{1}, \ldots, k_{l}$.

The basic tool in the study of layered permutations is the following theorem of Stromquist. We give a proof which is somewhat shorter than the proof in [1], both for its own interest and because we will reuse the method of proof when studying clumpy and layered patterns.

Theorem 4 Let $\Pi \subset S_{m}$ be a set of layered permutations. Among all permutations $\sigma \in S_{n}$ which maximize $\nu(\Pi, \sigma)$, there exists one which is layered.

Proof. Given a permutation $\sigma \in S_{n}$, we represent $\sigma$ by its graph $\{(i, \sigma(i)) \mid 1 \leq i \leq n\}$. More generally, any set of $n$ points $P=\left\{\left(x_{1}, y_{1}\right), \ldots,\left(x_{n}, y_{n}\right)\right\}$ with distinct $x$-coordinates and distinct $y$-coordinates defines a permutation in $S_{n}$, namely the permutation orderisomorphic to $y_{1} y_{2} \cdots y_{n}$ if we label the points so that $x_{1}<x_{2}<\cdots<x_{n}$. This permutation is layered if and only if the set $P$ has the following property: for any two points $u$, $v \in P$ with $u$ above and to the left of $v$, if $u$ and $v$ have consecutive $x$-coordinates, then they also have consecutive $y$-coordinates. We will work with permutations in this planar representation, so we will write $\nu(\Pi, P)$ for $\nu(\Pi, \sigma)$ where $\sigma \in S_{n}$ is the permutation corresponding to $P$.

Let $P$ be a planar set with $n$ points which maximizes $\nu(\Pi, P)$. We will show that we can make a series of moves of points of $P$, preserving $\nu(\Pi, P)$, such that the resulting set $P^{\prime}$ is layered. Suppose $u$ and $v$ are points of $P$ with consecutive $x$-coordinates such that $u$ lies above and to the left of $v$. Consider the two sets $P_{1}$ and $P_{2}$ obtained from $P$ by the following moves: to obtain $P_{1}$, we move $v$ up to be just below $u$; to obtain $P_{2}$, we move $u$ down to be just above $v$. In each case, $u$ and $v$ have the same order relation as before the move ( $u$ is still above and to the left of $v$ ), and after the move, $u$ and $v$ have identical order relation to every other point $w$. See Figure 1.

An occurrence of $\Pi$ in $P$ is a subset of $m$ points of $P$ which corresponds to a permutation $\pi \in \Pi$. We classify these occurrences into four types; those which contain neither $u$ nor $v$, those which contain $u$ but not $v$, those which contain $v$ but not $u$, and those which contain both $u$ and $v$. Denote the number of occurrences of each of these types by $\nu(\Pi, P, \bar{u}, \bar{v}), \nu(\Pi, P, u, \bar{v}), \nu(\Pi, P, \bar{u}, v)$, and $\nu(\Pi, P, u, v)$, respectively; then

$$
\nu(\Pi, P)=\nu(\Pi, P, \bar{u}, \bar{v})+\nu(\Pi, P, u, \bar{v})+\nu(\Pi, P, \bar{u}, v)+\nu(\Pi, P, u, v) .
$$




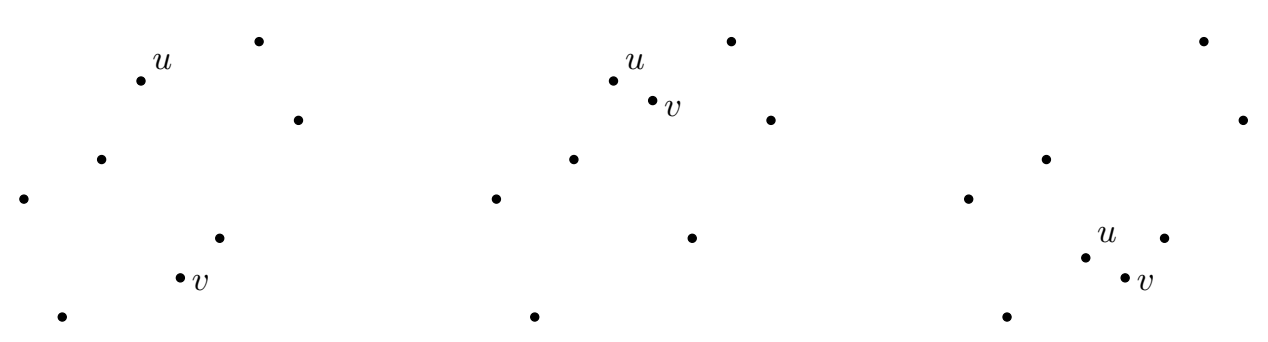

Figure 1: The planar sets $P, P_{1}$, and $P_{2}$

Let us now consider $\nu\left(\Pi, P_{1}\right)$ and $\nu\left(\Pi, P_{2}\right)$. Every occurrence of $\Pi$ in $P$ not containing $v$ corresponds to an occurrence in $P_{1}$ not containing $v$, so $\nu\left(\Pi, P_{1}, \bar{u}, \bar{v}\right)=\nu(\Pi, P, \bar{u}, \bar{v})$ and $\nu\left(\Pi, P_{1}, u, \bar{v}\right)=\nu(\Pi, P, u, \bar{v})$. In $P_{1}, u$ and $v$ have the same order relation to every other point, so $\nu\left(\Pi, P_{1}, \bar{u}, v\right)=\nu\left(\Pi, P_{1}, u, \bar{v}\right)=\nu(\Pi, P, u, \bar{v})$. Finally, consider an occurrence of $\Pi$ in $P$ which contains both $u$ and $v$, in other words, a subset $Q \subset P$ containing $u$ and $v$ such that $Q$ is isomorphic to a permutation $\pi \in \Pi$. Since $u$ and $v$ have consecutive $x$-coordinates in $P$, they must correspond to two consecutive letters in $\pi$, say $\pi(i)$ and $\pi(i+1)$. Also, since $u$ is above and to the left of $v$ in $P$, we have $\pi(i)>\pi(i+1)$. Now $\pi$ is layered, so $\pi(i)$ and $\pi(i+1)$ are consecutive letters in the same layer of $\pi$; therefore there is no letter $\pi(j)$ in $\pi$ with $\pi(i)>\pi(j)>\pi(i+1)$, so there is no point $w \in Q$ with $y$-coordinate between those of $u$ and $v$. Since the new $y$-coordinate of $v$ in $P_{1}$ is in this range, the order relation of $v$ to every other vertex $w \in Q$ is unchanged, so $Q$ is also an occurrence of $\Pi$ in $P_{1}$. Therefore $\nu\left(\Pi, P_{1}, u, v\right) \geq \nu(\Pi, P, u, v)$. So considering the total

$$
\nu\left(\Pi, P_{1}\right)=\nu\left(\Pi, P_{1}, \bar{u}, \bar{v}\right)+\nu\left(\Pi, P_{1}, u, \bar{v}\right)+\nu\left(\Pi, P_{1}, \bar{u}, v\right)+\nu\left(\Pi, P_{1}, u, v\right),
$$

we obtain

$$
\nu\left(\Pi, P_{1}\right) \geq \nu(\Pi, P, \bar{u}, \bar{v})+2 \nu(\Pi, P, u, \bar{v})+\nu(\Pi, P, u, v) .
$$

Similarly,

$$
\nu\left(\Pi, P_{2}\right) \geq \nu(\Pi, P, \bar{u}, \bar{v})+2 \nu(\Pi, P, \bar{u}, v)+\nu(\Pi, P, u, v) .
$$

Adding the last two inequalities, we obtain

$$
\begin{aligned}
\nu\left(\Pi, P_{1}\right)+\nu\left(\Pi, P_{2}\right) \geq & 2 \nu(\Pi, P, \bar{u}, \bar{v})+2 \nu(\Pi, P, u, \bar{v}) \\
& +2 \nu(\Pi, P, \bar{u}, v)+2 \nu(\Pi, P, u, v) \\
= & 2 \nu(\Pi, P) .
\end{aligned}
$$

But $P$ was chosen to maximize $\nu(\Pi, P)$; therefore $\nu\left(\Pi, P_{1}\right)$ and $\nu\left(\Pi, P_{2}\right)$ are each less than or equal to $\nu(\Pi, P)$. So equality must hold and we obtain $\nu\left(\Pi, P_{1}\right)=\nu\left(\Pi, P_{2}\right)=\nu(\Pi, P)$. Hence $P_{1}$ and $P_{2}$ are also $\Pi$-maximizing sets. Thus we have shown that if $P$ is a planar set maximizing $\nu(\Pi, P)$ and $u$ and $v$ are points in $P$ with consecutive $x$-coordinates such that $u$ lies above and to the left of $v$, then we may move $v$ to just below $u$ or $u$ to just above $v$ and in either case the $\Pi$-maximality is preserved.

Now let $P$ be a planar set which maximizes $\nu(\Pi, P)$, and consider all points in $P$ which are higher than any point to the left. Label these points $u_{1}, \ldots, u_{k}$, in increasing order of 
$x$-coordinate; then $u_{1}$ is the leftmost point of $P$, and $u_{i+1}$ lies above and to the right of $u_{i}$ for each $i$. Now, from left to right, move each point $v$ which is not among the $u_{i}$ to be just less than the point immediately to its left. For each $i$, this process will move the points with $x$-coordinate between those of $u_{i}$ and $u_{i+1}$ to form a decreasing sequence starting at $u_{i}$ with all its points higher than any point before $u_{i}$. Thus, after performing this process for each $i=1, \ldots, k$, the resulting planar set $P^{\prime}$ is layered, and $\nu\left(\Pi, P^{\prime}\right)=\nu(\Pi, P)$, so $P^{\prime}$ is a П-maximizer, proving the theorem.

We define layered patterns by analogy with layered permutations.

Definition 6 A layered pattern is a strictly increasing sequence of nonincreasing segments, in other words, a pattern which can be written $\pi=\pi_{1} \pi_{2} \cdots \pi_{l}$ where $\pi_{1}<\pi_{2}<$ $\cdots<\pi_{l}$ and each $\pi_{i}$ is a nonincreasing sequence. The segments $\pi_{i}$ are called the layers of $\pi$.

A proof of the following conjecture would allow us to compute $d(\pi)$ for many layered patterns $\pi$.

Conjecture 5 ([2, Conjecture 2.8]) If $\Pi$ is a set of layered patterns, then among all $\Pi$-maximizers $\sigma \in[k]^{n}$, there exists one which is layered.

As a first step in this direction, we have the following definition and result.

Definition 7 A pattern $\pi \in \Sigma^{n}$ is clumpy if for any letter $x \in \Sigma$, all occurrences of $x$ in $\pi$ are consecutive. Equivalently, if the $i$ th and $j$ th letters of $\pi$ are both $x$, then all letters of $\pi$ between the $i$ th and the $j$ th are $x$ as well.

Theorem 6 Let $\Pi$ be a set of clumpy patterns. Among all words $\sigma \in[k]^{n}$ which maximize $\nu(\Pi, \sigma)$, there exists one which is clumpy.

Proof. Let $\sigma \in[k]^{n}$ be a word which maximizes $\nu(\Pi, \sigma)$. Suppose that some letter $x$ appears more than once in $\sigma$. Consider two particular appearances of $x$ in $\sigma$; write $\sigma=$ $\tau_{1} x_{1} \tau_{2} x_{2} \tau_{3}$, where the $\tau_{i}$ are words on the alphabet $[k]$, and we label the two appearances of $x$ in question $x_{1}$ and $x_{2}$ for ease of reference. We divide the occurrences of $\Pi$ in $\sigma$ into four types according to whether they contain $x_{1}$ and $x_{2}$; using notation analogous to that used in the proof of Theorem 4, we have

$$
\nu(\Pi, \sigma)=\nu\left(\Pi, \sigma, \bar{x}_{1}, \bar{x}_{2}\right)+\nu\left(\Pi, \sigma, x_{1}, \bar{x}_{2}\right)+\nu\left(\Pi, \sigma, \bar{x}_{1}, x_{2}\right)+\nu\left(\Pi, \sigma, x_{1}, x_{2}\right) .
$$

Now consider the two new words

$$
\sigma_{1}=\tau_{1} x_{1} x_{2} \tau_{2} \tau_{3}, \quad \sigma_{2}=\tau_{1} \tau_{2} x_{1} x_{2} \tau_{3}
$$

Let us compute $\nu\left(\Pi, \sigma_{1}\right)$. Since the only difference between $\sigma$ and $\sigma_{1}$ is the location of $x_{2}$, we have $\nu\left(\Pi, \sigma_{1}, \bar{x}_{1}, \bar{x}_{2}\right)=\nu\left(\Pi, \sigma, \bar{x}_{1}, \bar{x}_{2}\right)$ and $\nu\left(\Pi, \sigma_{1}, x_{1}, \bar{x}_{2}\right)=\nu\left(\Pi, \sigma, x_{1}, \bar{x}_{2}\right)$. Also, the letters $x_{1}$ and $x_{2}$ are consecutive in $\sigma_{1}$ and equal, so $\nu\left(\Pi, \sigma_{1}, \bar{x}_{1}, x_{2}\right)=\nu\left(\Pi, \sigma_{1}, x_{1}, \bar{x}_{2}\right)=$ 
$\nu\left(\Pi, \sigma, x_{1}, \bar{x}_{2}\right)$. Finally, consider an occurrence of $\pi \in \Pi$ in $\sigma$ containing both $x_{1}$ and $x_{2}$. We can write $\pi=\pi_{1} y_{1} \pi_{2} y_{2} \pi_{3}$, where $\pi_{i}$ is the part of $\pi$ which occurs in $\tau_{i}$ and the letter $y_{i}$ corresponds to $x_{i}$. Since $x_{1}$ and $x_{2}$ are the same letter, $y_{1}$ and $y_{2}$ are equal, say $y_{1}=y_{2}=y$; and since $\pi$ is clumpy, every letter of $\pi_{2}$ is $y$, so $\pi_{2}=y^{j}$ for some $j$. Therefore taking the corresponding letters in $\sigma_{1}$ gives an occurrence of $\pi_{1} y_{1} y_{2} \pi_{2} \pi_{3}=\pi_{1} y^{j+2} \pi_{3}=\pi$, so $\nu\left(\Pi, \sigma_{1}, x_{1}, x_{2}\right) \geq \nu\left(\Pi, \sigma, x_{1}, x_{2}\right)$. It follows that

$$
\nu\left(\Pi, \sigma_{1}\right) \geq \nu\left(\Pi, \sigma, \bar{x}_{1}, \bar{x}_{2}\right)+2 \nu\left(\Pi, \sigma, x_{1}, \bar{x}_{2}\right)+\nu\left(\Pi, \sigma, x_{1}, x_{2}\right) .
$$

Similarly,

$$
\nu\left(\Pi, \sigma_{2}\right) \geq \nu\left(\Pi, \sigma, \bar{x}_{1}, \bar{x}_{2}\right)+2 \nu\left(\Pi, \sigma, \bar{x}_{1}, x_{2}\right)+\nu\left(\Pi, \sigma, x_{1}, x_{2}\right) .
$$

Adding these yields $\nu\left(\Pi, \sigma_{1}\right)+\nu\left(\Pi, \sigma_{2}\right) \geq 2 \nu(\Pi, \sigma)$; since $\sigma$ was chosen such that $\nu(\Pi, \sigma)$ was maximal, we must have equality, so $\nu\left(\Pi, \sigma_{1}\right)=\nu\left(\Pi, \sigma_{2}\right)=\nu(\Pi, \sigma)$. Thus we have shown that we may move together any two equal letters of $\sigma$ and preserve the maximality of $\nu(\Pi, \sigma)$. By repeating this process we may move all equal letters into blocks, giving us a clumpy pattern $\sigma^{\prime} \in[k]^{n}$ with $\nu\left(\Pi, \sigma^{\prime}\right)$ maximal.

Note that any layered pattern $\pi$ is clumpy, because any given letter $x$ can appear in at most one layer $\pi_{i}$, and $\pi_{i}$ is nonincreasing so all occurrences of $x$ in $\pi_{i}$ are consecutive. Therefore, if $\Pi$ is a set of layered patterns, then among all $\Pi$-maximizers $\sigma \in[k]^{n}$, there exists one which is clumpy.

We can think of clumpy patterns as "weighted permutations" as follows: If $\pi \in[l]^{m}$ is a clumpy pattern, then we can write $\pi=\pi_{1}^{a_{1}} \cdots \pi_{l}^{a_{l}}$ where $\pi_{1} \cdots \pi_{l}$ is a permutation of $[l]$ and the $a_{i}$ are nonnegative integers. We call $\pi_{1} \cdots \pi_{l}$ the underlying permutation of $\pi$. By the previous result, when computing $d(\pi, k, n)$, we need only consider words $\sigma \in[k]^{n}$ which are clumpy. If we write $\sigma$ as a weighted permutation $\sigma=\sigma_{1}^{b_{1}} \cdots \sigma_{k}^{b_{k}}$, then we can count occurrences of $\pi$ in $\sigma$ by the corresponding occurrences of the permutation $\bar{\pi}=\pi_{1} \cdots \pi_{l}$ in $\bar{\sigma}=\sigma_{1} \cdots \sigma_{l}$; more precisely, we have

$$
\nu(\pi, \sigma)=\sum\left(\begin{array}{l}
b_{i_{1}} \\
a_{1}
\end{array}\right) \cdots\left(\begin{array}{l}
b_{i_{l}} \\
a_{l}
\end{array}\right)
$$

where the sum is taken over all subsequences $\sigma_{i_{1}} \cdots \sigma_{i_{l}}$ of $\bar{\sigma}$ isomorphic to $\bar{\pi}$. The difficulty in extending Theorem 4 to layered patterns seems to lie in the fact that a single weight $b_{j}$ may appear in the sum above as $\left(\begin{array}{l}b_{j} \\ a_{i}\end{array}\right)$ with different $a_{i}$ in different terms. Indeed, when the values $a_{i}$ are all equal, we can prove that Conjecture 5 holds.

Theorem 7 Let $\bar{\Pi}$ be a set of layered permutations, and let $\Pi$ be the set of patterns obtained by repeating each letter of each permutation $\bar{\pi} \in \bar{\Pi} r$ times. Then among all $\Pi$-maximizers $\sigma \in[k]^{n}$, there exists one which is layered.

Proof. By Theorem 6, we only need to consider words $\sigma \in[k]^{n}$ which are clumpy. So let $\sigma \in[k]^{n}$ be a clumpy П-maximizer. Write $\sigma=\sigma_{1}^{b_{1}} \cdots \sigma_{k}^{b_{k}}$ where $\bar{\sigma}=\sigma_{1} \cdots \sigma_{k}$ is a permutation of $[k]$ and the $b_{i}$ are nonnegative integers. Let $P$ be the planar set 
corresponding to $\bar{\sigma}$ as in the proof of Theorem 4 , and assign the weight $b_{i}$ to each point $\left(i, \sigma_{i}\right)$. We call this the weighted planar set corresponding to the clumpy word $\sigma$.

We first use induction on $k$ to remove all points with weight less than $r$. If $k=1$, then $\sigma$ is automatically layered, so suppose $k>1$ and assume the theorem holds for $k-1$. Suppose some point $\left(i, \sigma_{i}\right)$ has weight $b_{i}$ less than $r$. Since every letter in any pattern $\pi \in \Pi$ is repeated $r$ times, there is no occurrence of $\pi$ in $\sigma$ which uses the letters $\sigma_{i}^{b_{i}}$. So removing these letters and adding $b_{i}$ to the weight of another letter in $\sigma$ yields a word $\sigma^{\prime}$ on an alphabet of $k-1$ letters with at least as many occurrences of $\Pi$ as $\sigma$. By the induction hypothesis, there exists a layered word $\sigma^{\prime \prime}$ in $[k-1]^{n}$ with at least as many occurrences of $\Pi$ as $\sigma^{\prime}$. So $\nu\left(\Pi, \sigma^{\prime \prime}\right) \geq \nu(\Pi, \sigma)$, and $\sigma^{\prime \prime} \in[k-1]^{n} \subset[k]^{n}$ is a layered $\Pi$-maximizer, as desired. Therefore we may assume that every point $\left(i, \sigma_{i}\right)$ has weight at least $r$.

Now we emulate the proof of Theorem 4. Suppose $u$ and $v$ are points of $P$ with consecutive $x$-coordinates such that $u$ lies above and to the left of $v$. We form new planar sets $P_{1}, P_{2}$ as before, $P_{1}$ by moving $v$ up to just below $u$, and $P_{2}$ by moving $u$ down to just above $v$. Then we write

$$
\nu(\Pi, P)=\nu(\Pi, P, \bar{u}, \bar{v})+\nu(\Pi, P, u, \bar{v})+\nu(\Pi, P, \bar{u}, v)+\nu(\Pi, P, u, v) .
$$

As in Theorem 4, we have

$$
\begin{gathered}
\nu\left(\Pi, P_{1}, \bar{u}, \bar{v}\right)=\nu(\Pi, P, \bar{u}, \bar{v}), \quad \nu\left(\Pi, P_{1}, u, \bar{v}\right)=\nu(\Pi, P, u, \bar{v}), \\
\text { and } \quad \nu\left(\Pi, P_{1}, u, v\right) \geq \nu(\Pi, P, u, v) .
\end{gathered}
$$

However, in general $\nu\left(\Pi, P_{1}, \bar{u}, v\right) \neq \nu\left(\Pi, P_{1}, u, \bar{v}\right)$, because the points $u$ and $v$ may not have equal weight. Instead, writing $|u|$ and $|v|$ for the weights of points $u$ and $v$, we have $\nu\left(\Pi, P_{1}, \bar{u}, v\right)=\frac{\left(\begin{array}{c}|v| \\ r\end{array}\right)}{\left(\begin{array}{c}|u| \\ r\end{array}\right)} \nu\left(\Pi, P_{1}, u, \bar{v}\right)$, because any occurrence of a pattern $\pi \in \Pi$ counted by $\nu\left(\Pi, P_{1}, u, \bar{v}\right)$ uses exactly $r$ of the $|u|$ letters at the point $u$, so we can group these into sets of size $\left(\begin{array}{c}|u| \\ r\end{array}\right)$, each of which corresponds to a set of $\left(\begin{array}{c}|v| \\ r\end{array}\right)$ occurrences in $\nu\left(\Pi, P_{1}, \bar{u}, v\right)$. (Note that since $|u| \geq r$, the denominator $\left(\begin{array}{c}|u| \\ r\end{array}\right)$ is nonzero.) Therefore we have

$$
\nu\left(\Pi, P_{1}\right) \geq \nu(\Pi, P, \bar{u}, \bar{v})+\nu(\Pi, P, u, \bar{v})+\frac{\left(\begin{array}{c}
|v| \\
r
\end{array}\right)}{\left(\begin{array}{c}
|u| \\
r
\end{array}\right)} \nu(\Pi, P, u, \bar{v})+\nu(\Pi, P, \bar{u}, \bar{v}) .
$$

Similarly

$$
\nu\left(\Pi, P_{2}\right) \geq \nu(\Pi, P, \bar{u}, \bar{v})+\frac{\left(\begin{array}{c}
|u| \\
r
\end{array}\right)}{\left(\begin{array}{c}
|v| \\
r
\end{array}\right)} \nu(\Pi, P, \bar{u}, v)+\nu(\Pi, P, \bar{u}, v)+\nu(\Pi, P, \bar{u}, \bar{v}) .
$$

Multiply the first inequality by $\left(\begin{array}{c}|u| \\ r\end{array}\right)$, the second by $\left(\begin{array}{c}|v| \\ r\end{array}\right)$, and add; we obtain $\left(\begin{array}{c}|u| \\ r\end{array}\right) \nu\left(\Pi, P_{1}\right)+$ $\left(\begin{array}{c}|v| \\ r\end{array}\right) \nu\left(\Pi, P_{2}\right) \geq\left(\left(\begin{array}{c}|u| \\ r\end{array}\right)+\left(\begin{array}{c}|v| \\ r\end{array}\right)\right) \nu(\Pi, P)$. Since $\sigma$ is a $\Pi$-maximizer, it follows that $\nu\left(\Pi, P_{1}\right)=$ $\nu\left(\Pi, P_{2}\right)=\nu(\Pi, P)$. Therefore $P_{1}$ and $P_{2}$ are both $\Pi$-maximizing planar sets, and we finish the proof as in Theorem 4. So there exists a layered $\Pi$-maximizer $\sigma \in[k]^{n}$. 


\section{The patterns $1^{p} 3^{q} 2^{r}$}

We can think of a clumpy pattern $\pi=\pi_{1}^{a_{1}} \cdots \pi_{l}^{a_{l}} \in[l]^{m}$ as a weighted version of its underlying permutation $\bar{\pi}=\pi_{1} \cdots \pi_{l}$. When $\bar{\pi}$ is the identity $12 \ldots l$, the pattern $\pi$ is nondecreasing. This case was handled in [2], where the following result is proved.

Proposition 8 Define a one-to-one correspondence between nondecreasing patterns of length $n$ and layered permutations of length $n$ by

nondecreasing pattern $\pi=1^{a_{1}} \cdots l^{a_{l}} \leftrightarrow$ layered permutation $\hat{\pi}=\left[a_{1}, \ldots, a_{l}\right]$.

Then for any $\pi$ and $\sigma, \nu(\pi, \sigma)=\nu(\hat{\pi}, \hat{\sigma})$, and there is a nondecreasing $\pi$-maximizer in $[n]^{n}$. In particular, $\delta(\pi)=\delta(\hat{\pi})$.

Thus the packing density problem for such patterns can be reduced to the case of layered permutations. It is natural to next consider other short permutations $\bar{\pi}$. Since packing density is invariant under the symmetries of reversal $(\pi(i) \rightarrow \pi(m-i+1))$ and complement $(\pi(i) \rightarrow l-\pi(i)+1)$, there is only one other case with $l \leq 3$, namely $\bar{\pi}=132$. We will show here that Conjecture 5 holds for these patterns.

Theorem 9 Let $\Pi=\left\{1^{p} 3^{q} 2^{r}\right\}$, where $p, q, r \geq 1$. Among all $\Pi$-maximizers $\sigma \in[k]^{n}$, there exists one which is layered.

Proof. First we show that we may assume $q \geq r$. If $\pi=\pi_{1}^{a_{1}} \cdots \pi_{l}^{a_{l}}$ is a clumpy pattern where $\bar{\pi}=\pi_{1} \cdots \pi_{l} \in S_{l}$, let $\pi_{1}^{\prime} \cdots \pi_{l}^{\prime}=\bar{\pi}^{-1}$ and define $\pi^{-1}=\pi_{1}^{\prime} a_{1}^{\prime} \cdots \pi_{l}^{\prime} a_{\pi_{l}^{\prime}}$; in graphical terms, $\pi^{-1}$ is obtained from the weighted planar set corresponding to $\pi$ by reflection in the line $x=y$. (The point $\left(i, \pi_{i}\right)$ with weight $a_{i}$ reflects to the point $\left(\pi_{i}, i\right)$ with weight $a_{i}$, so for each $j=1, \ldots, n$ we get the point $\left(j, \pi_{j}^{\prime}\right)$ with weight $a_{\pi_{j}^{\prime}}$.) The graphical interpretation makes it clear that if $\pi$ and $\sigma$ are clumpy then $\nu(\pi, \sigma)=\nu\left(\pi^{-1}, \sigma^{-1}\right)$. Since the inverse of a layered word in $[k]^{n}$ is another layered word in $[k]^{n}$, we can replace $\pi=1^{p} 3^{q} 2^{r}$ by $\pi^{-1}=1^{p} 3^{r} 2^{q}$ in the statement of the theorem. Thus we may assume without loss of generality that $q \geq r$.

Given a clumpy word $\sigma=\sigma_{1}^{b_{1}} \cdots \sigma_{k}^{b_{k}}$, where the $\sigma_{i}$ are distinct and the $b_{i}$ are positive integers, call the (unordered) partition $n=b_{1}+\cdots+b_{k}$ the weight set of $\sigma$. We will prove the following statement by induction on $k$ :

Let $\sigma \in[k]^{n}$ be any clumpy word. Then there is a layered word $\sigma^{\prime} \in[k]^{n}$ with the same weight set as $\sigma$ such that $\nu\left(\Pi, \sigma^{\prime}\right) \geq \nu(\Pi, \sigma)$.

For $k \leq 2$ the claim is trivial, since $\nu(\Pi, \sigma)=0$ for all $\sigma \in[k]^{n}$. So assume $k \geq 3$ and let $\sigma \in[k]^{n}$ be a clumpy word. If some letter in $[k]$ does not appear in $\sigma$, then $\sigma$ is order-isomorphic to a word in $[k-1]^{n}$ and we can apply the inductive hypothesis. So assume that every letter of $[k]$ appears in $\sigma$, that is, $\sigma$ is a weighted version of some permutation $\bar{\sigma} \in S_{k}$. Let $\Sigma \subset[k]^{n}$ be the set of all clumpy words in $[k]^{n}$ with the same weight set as $\sigma$. Then we may assume that $\nu(\Pi, \sigma)$ is maximal among all $\sigma \in \Sigma$. We will say that $\sigma$ is a $\Pi$-maximizer in $\Sigma$, since $\sigma$ might not be a $\Pi$-maximizer in all of $[k]^{n}$. Let $P$ 
be the weighted planar set corresponding to $\sigma$. Consider the topmost point $u_{1}$ of $P$. If $u_{1}$ has weight less than $q$, then there cannot be any occurrences of $1^{p} 3^{q} 2^{r}$ in $P$ using $u_{1}$, so by removing the point $u_{1}$ we obtain a word $\sigma^{\prime} \in[k-1]^{n-\left|u_{1}\right|}$ with as many $\Pi$-occurrences as $\sigma$. By the inductive hypothesis we may replace $\sigma^{\prime}$ by a layered word with at least as many $\Pi$-occurrences and the same weight set. Now add a point $u_{1}^{\prime}=(k, k)$ with weight $\left|u_{1}\right|$; this gives a layered word in $[k]^{n}$ with at least as many $\Pi$-occurrences and the same weight set as $\sigma$. Therefore we will assume henceforth that $u_{1}$ has weight at least $q$.

Now suppose $\sigma$ is a $\Pi$-maximizer in $\Sigma$ with weighted planar set $P$ and there is a sequence of points $u_{1}, \ldots, u_{j}$ such that

- $u_{1}$ is the topmost point of $P$ and has weight at least $q$;

- for each $2 \leq i \leq j, u_{i}$ lies immediately below and to the right of $u_{i-1}$.

Call such a sequence of points a chain of length $j$. First, suppose $u_{j}$ is the rightmost point of $P$. Then the points $u_{1}, \ldots, u_{j}$ form a decreasing sequence above and to the right of all other points of $P$. Let $\sigma_{1}$ be the word corresponding to the points $u_{1}, \ldots, u_{j}$ and let $\sigma_{0}$ be the word corresponding to the other points of $P$; then $\nu(\Pi, \sigma)=\nu\left(\Pi, \sigma_{0}\right)+$ $\nu\left(1^{p}, \sigma_{0}\right) \nu\left(3^{q} 2^{r}, \sigma_{1}\right)$. By the inductive hypothesis we may replace $\sigma_{0}$ by a layered word $\sigma_{0}^{\prime}$ with the same weight set as $\sigma_{0}$ such that $\nu\left(\Pi, \sigma_{0}^{\prime}\right) \geq \nu\left(\Pi, \sigma_{0}\right)$. Since $\sigma_{0}$ and $\sigma_{0}^{\prime}$ have the same weight set, $\nu\left(1^{p}, \sigma_{0}\right)=\nu\left(1^{p}, \sigma_{0}^{\prime}\right)$. Therefore the word $\sigma^{\prime}=\sigma_{0}^{\prime} \sigma_{1}$ has $\nu\left(\Pi, \sigma^{\prime}\right)=$ $\nu\left(\Pi, \sigma_{0}^{\prime}\right)+\nu\left(1^{p}, \sigma_{0}^{\prime}\right) \nu\left(3^{q} 2^{r}, \sigma_{1}\right) \geq \nu(\Pi, \sigma)$. Now $\sigma^{\prime}$ is a layered word with the same weight set as $\sigma$, so the claim holds.

We now show that if $\sigma$ is a $\Pi$-maximizer in $\Sigma$ with weighted planar set $P$ containing a chain $u_{1}, \ldots, u_{j}$, and $u_{j}$ is not the rightmost point of $P$, then we can find a $\Pi$-maximizer $\sigma^{\prime} \in \Sigma$ whose weighted planar set $P^{\prime}$ contains a chain of length $j+1$. Introduce the following notation: If $P$ is a weighted planar set and $U, V, W$ are subsets, let $\nu_{132}(U, V, W)$ be the number of occurrences of $1^{p} 3^{q} 2^{r}$ in $P$ such that 1 corresponds to a point $u \in U$, 2 corresponds to a point $v \in V$, and 3 corresponds to a point $w \in W$. Symbolically, we have

$$
\nu_{132}(U, V, W)=\sum\left(\begin{array}{c}
|u| \\
p
\end{array}\right)\left(\begin{array}{c}
|v| \\
q
\end{array}\right)\left(\begin{array}{c}
|w| \\
r
\end{array}\right)
$$

where the sum is taken over all triplets of points $u \in U, v \in V, w \in W$ such that $u_{x}<v_{x}<w_{x}$ and $u_{y} v_{y} w_{y}$ is order-isomorphic to 132 . Here we use $u_{x}$ and $u_{y}$ to denote the $x$ - and $y$-coordinates of a point $u$ and $|u|$ to denote its weight. Similarly we will write $\nu_{13}(U, V)$, for example, to denote the sum of $\left(\begin{array}{c}|u| \\ p\end{array}\right)\left(\begin{array}{c}|v| \\ q\end{array}\right)$ over pairs $u \in U, v \in V$ with $u$ below and to the left of $v$, etc. (The notation $\nu_{132}, \nu_{13}, \ldots$ should be regarded as shorthands for the unwieldy but more precise $\nu_{1^{p} 3^{q} 2^{r}}, \nu_{1^{p} 3^{q}}, \ldots$; in general 1 represents $1^{p}$, 3 represents $3^{q}$, and 2 represents $2^{r}$.) For example, if $P$ is the planar set corresponding to a clumpy word $\sigma$, then $\nu_{132}(P, P, P)=\nu(\Pi, \sigma)$, and $\nu_{13}(P, P)=\nu\left(1^{p} 3^{q}, \sigma\right)$, etc.

Now let $\sigma$ be a $\Pi$-maximizer in $\Sigma$ whose weighted planar set $P$ contains a chain $u_{1}$, $\ldots, u_{j}$. Let $U=\left\{u_{1}, \ldots, u_{j}\right\}$, and let $v$ be the topmost point among the points of $P$ to the right of $U$. Suppose that $v$ is not the topmost point of $P \backslash U$; that is, the set $A$ of points in $P$ which lie above $v$ and to the left of $U$ is nonempty. We will show that moving 


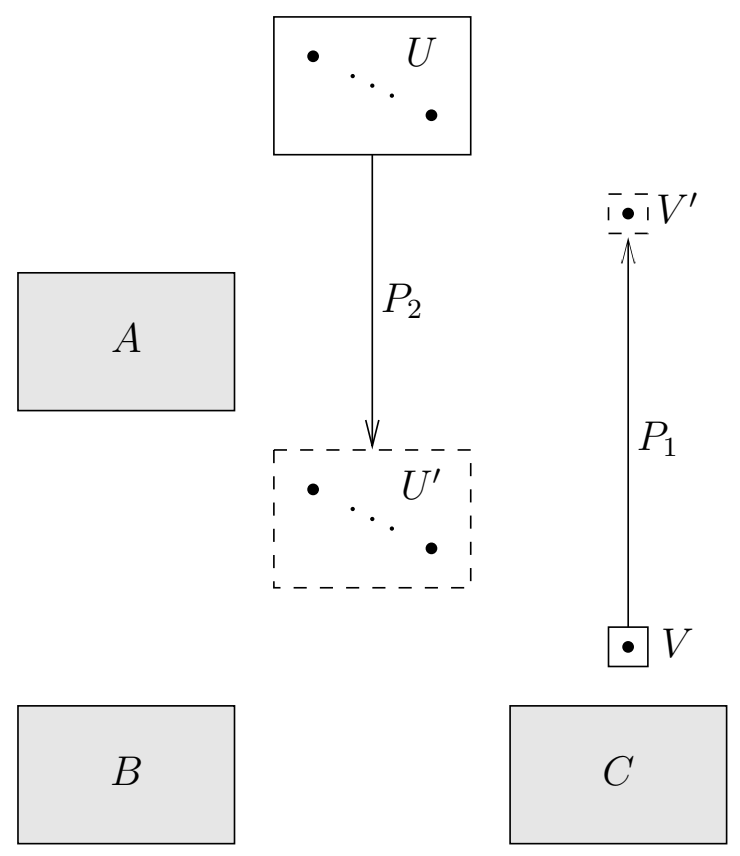

Figure 2: Lengthening a chain, part I

$v$ up to lie above $A$ but below $U$ yields another $\Pi$-maximizer $\sigma_{1}$ in $\Sigma$. Let $V=\{v\}$, let $B$ be the set of points in $P$ to the left of $U$ and below $v$, and let $C$ be the set of points in $P$ to the right of $U$ other than $v$ (all of which lie below $v$ ). Then $P=A \cup B \cup C \cup U \cup V$. Let $V^{\prime}=\left\{v^{\prime}\right\}$, where $v^{\prime}$ is a copy of $v$, directly above $v$, and above $A$ but below $U$; and let $U^{\prime}$ be a copy of $U$, directly below $U$, and below $A$ but above $v$. See Figure 2 . Set

$$
P_{1}=A \cup B \cup C \cup U \cup V^{\prime}, \quad P_{2}=A \cup B \cup C \cup U^{\prime} \cup V .
$$

Consider the quantity $\nu\left(\Pi, P_{1}\right)-\nu(\Pi, P)$. We can expand $\nu(\Pi, P)$ as the sum of the terms $\nu_{132}(X, Y, Z)$ over all $X, Y, Z \in\{A, B, C, U, V\}$. When we subtract this sum from the corresponding expression for $\nu\left(\Pi, P_{1}\right)$, all of the $\nu_{132}$ terms not involving $V$ or $V^{\prime}$ will cancel. Furthermore, since $V$ and $V^{\prime}$ have different relative position only to $A$, all terms not involving $A$ will cancel. Finally, we can see in Figure 2 that all but two of the terms involving both $A$ and $V$ or $V^{\prime}$ are zero, so

$$
\nu\left(\Pi, P_{1}\right)-\nu(\Pi, P)=\nu_{132}\left(A, U, V^{\prime}\right)-\nu_{132}(B, A, V) .
$$

Similarly, in $P_{2}$ the only change is in the positions of $U$ and $U^{\prime}$ relative to $V$, so

$$
\nu\left(\Pi, P_{2}\right)-\nu(\Pi, P)=\nu_{132}\left(B, A, U^{\prime}\right)-\nu_{132}(A, U, U) .
$$

We want $P_{1}$ to be a П-maximizer as well, so assume for the sake of contradiction that $\nu\left(\Pi, P_{1}\right)<\nu(\Pi, P)$. Since $\nu_{132}\left(A, U, V^{\prime}\right)-\nu_{132}(B, A, V)=\left(\nu_{13}(A, U)-\nu_{13}(B, A)\right) \nu_{2}\left(V^{\prime}\right)$, 


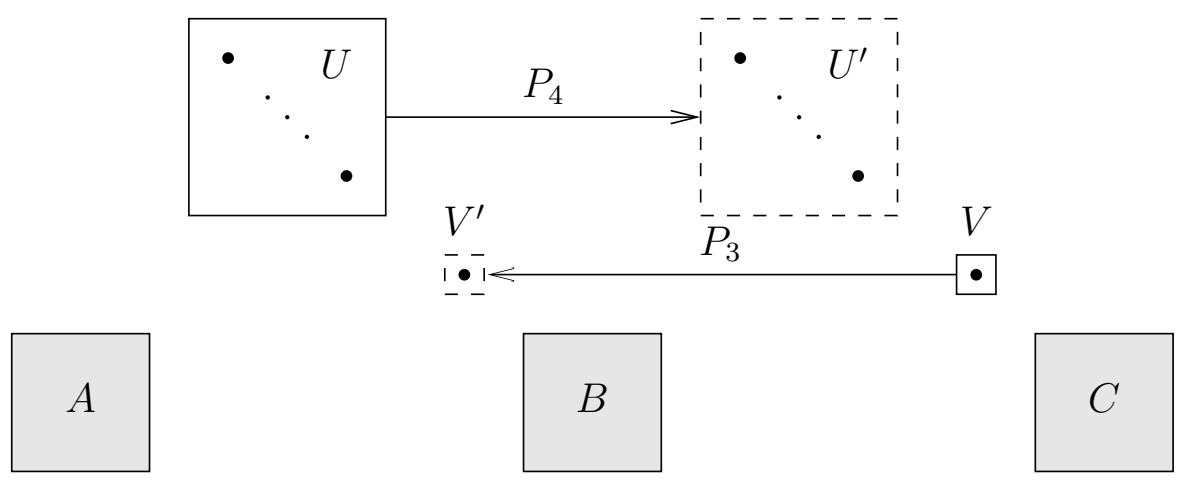

Figure 3: Lengthening a chain, part II

we conclude that $\nu_{13}(A, U)<\nu_{13}(B, A)$. Now observe that

$$
\begin{aligned}
\nu\left(\Pi, P_{2}\right)-\nu(\Pi, P) & =\nu_{132}\left(B, A, U^{\prime}\right)-\nu_{132}(A, U, U) \\
\geq & \left(\nu_{13}(B, A)-\nu_{13}(A, U)\right) \nu_{2}(U) \\
& >0
\end{aligned}
$$

because $\nu_{13}(B, A)>\nu_{13}(A, U)$ and $U$ has a point $u_{1}$ of weight $\left|u_{1}\right| \geq q \geq r$ so $\nu_{2}(U)>0$. This is a contradiction, as $P$ is a $\Pi$-maximizer. Therefore $\nu\left(\Pi, P_{1}\right) \geq \nu(\Pi, P)$, so $\sigma_{1}$ is a $\Pi$-maximizer as claimed. So if $v$ does not lie above all points to the left of $U$, moving it above these points yields another $\Pi$-maximizer in $\Sigma$. Hence we will assume that $v$ lies immediately below $u_{j}$.

Now we show by a similar argument that $v$ can be moved to lie immediately to the right of $u_{j}$. If $v$ does not already lie immediately to the right of $u_{j}$, then the set $B$ of points of $P$ which lie horizontally between $u_{j}$ and $v$ is nonempty. We will show that moving $v$ horizontally past $B$ yields another $\Pi$-maximizer $\sigma_{3} \in \Sigma$. Let $A$ be the set of points in $P$ to the left of $U$ and let $C$ be the set of points in $P$ to the right of $v$. Then $P=A \cup B \cup C \cup U \cup V$. Let $V^{\prime}=\left\{v^{\prime}\right\}$ where $v^{\prime}$ is a copy of $v$ moved horizontally to the left past $B$, and let $U^{\prime}$ be a copy of $U$ moved horizontally to the right past $B$. Set

$$
P_{3}=A \cup B \cup C \cup U \cup V^{\prime}, \quad P_{4}=A \cup B \cup C \cup U^{\prime} \cup V .
$$

From Figure 3 we see that

$$
\nu\left(\Pi, P_{3}\right)-\nu(\Pi, P)=\nu_{132}\left(A, V^{\prime}, B\right)-\nu_{132}(B, V, C)
$$

and

$$
\nu\left(\Pi, P_{4}\right)-\nu(\Pi, P)=\nu_{132}\left(B, U^{\prime}, U^{\prime}\right)+\nu_{132}\left(B, U^{\prime}, V\right)+\nu_{132}\left(B, U^{\prime}, C\right)-\nu_{132}(A, U, B) .
$$

Suppose that $P_{3}$ is not a $\Pi$-maximizer, so $\nu\left(\Pi, P_{3}\right)<\nu(\Pi, P)$. Since $\nu\left(\Pi, P_{3}\right)-\nu(\Pi, P)=$ $\nu_{132}\left(A, V^{\prime}, B\right)-\nu_{132}(B, V, C)=\left(\nu_{12}(A, B)-\nu_{12}(B, C)\right) \nu_{3}(V)$, we must have $\nu_{12}(A, B)<$ 
$\nu_{12}(B, C)$. Therefore

$$
\begin{aligned}
\nu\left(\Pi, P_{4}\right)-\nu(\Pi, P) & =\nu_{132}\left(B, U^{\prime}, U^{\prime}\right)+\nu_{132}\left(B, U^{\prime}, V\right)+\nu_{132}\left(B, U^{\prime}, C\right)-\nu_{132}(A, U, B) \\
& \geq\left(\nu_{12}(B, C)-\nu_{12}(A, B)\right) \nu_{3}(U) \\
& >0
\end{aligned}
$$

because $\nu_{12}(B, C)-\nu_{12}(A, B)>0$ and $\nu_{3}(U)>0$. This is a contradiction, so $P_{3}$ must be a П-maximizer. Now $P_{3}$ has a chain of length $j+1$, as desired.

To summarize the proof of the claim, we showed that if we start with a П-maximizer $\sigma$ in $\Sigma$, we may assume that $\sigma$ has topmost point $u_{1}$ with weight at least $q$. Then $u_{1}$ is a chain of length 1 in $\sigma$. If we have a $\Pi$-maximizer $\sigma$ with a chain of length $j$, then either the last point of the chain is the rightmost point of $\sigma$, and the claim follows from the inductive hypothesis, or we can find another $\Pi$-maximizer $\sigma^{\prime}$ with a chain of length $j+1$. Clearly the process of lengthening the chain must terminate at some point, proving the claim.

The statement of the theorem follows, because by Theorem 6 , there exists a clumpy $\Pi$-maximizer $\sigma \in[k]^{n}$, so by the claim, there is a layered $\Pi$-maximizer $\sigma^{\prime} \in[k]^{n}$.

\section{Computations of packing density}

The results of the preceding two sections describe the structure of $\pi$-maximizers for certain patterns $\pi$. Now we will use these tools to return to our original goal of computing values of $\delta(\pi)$. We will need the following results on simple permutations, which can be found in $[3]$.

Definition 8 A layered permutation $\pi$ with $l$ layers is simple if there is a sequence of permutations $\sigma_{n} \in S_{n}$ such that each permutation $\sigma_{n}$ has $l$ layers and $\lim _{n \rightarrow \infty} d\left(\pi, \sigma_{n}\right)=$ $\delta(\pi)$.

Proposition 10 If $\pi=\left[a_{1}, \ldots, a_{l}\right] \in S_{m}$ is a simple permutation, then

$$
\delta(\pi)=\left(\begin{array}{c}
m \\
a_{1}, \ldots, a_{l}
\end{array}\right) \frac{a_{1}^{a_{1}} \cdots a_{l}^{a_{l}}}{m^{m}} .
$$

Theorem 11 Let $\pi=\left[a_{1}, \ldots, a_{l}\right] \in S_{m}$. If $a_{i} \geq \log _{2}(l+1)$ for all $i$, then $\pi$ is a simple permutation.

We now show how the conclusion of Conjecture 5 can be used to bound $\delta(\pi)$ when $\pi$ is a layered pattern.

Proposition 12 Let $\pi=\pi_{1} \cdots \pi_{l}$ be a layered pattern, where $\pi_{1}<\cdots<\pi_{l}$ are the layers of $\pi$. Let $m_{i}$ be the length of $\pi_{i}$, and let $\rho=1^{m_{1}} \cdots l^{m_{l}}$. Suppose there exists an infinite sequence of layered words $\sigma^{(j)}$ of lengths $\left|\sigma^{(j)}\right| \rightarrow \infty$ such that $d\left(\pi, \sigma^{(j)}\right) \rightarrow \delta(\pi)$. (In particular, this condition holds if for every $n$ there is a layered $\pi$-maximizer in $[n]^{n}$.) Then

$$
\delta(\pi) \leq \delta(\rho) \delta\left(\pi_{1}\right) \cdots \delta\left(\pi_{l}\right)
$$


Proof. For convenience let $m=m_{1}+\cdots+m_{l}$ be the length of $\pi$, and write $\delta_{0}=$ $\delta(\rho) \delta\left(\pi_{1}\right) \cdots \delta\left(\pi_{l}\right)$. We first show that for any layered word $\sigma, \nu(\pi, \sigma) \leq \frac{|\sigma|^{m}}{m !} \delta_{0}$. Write $\sigma=\sigma_{1} \sigma_{2} \cdots \sigma_{k}$ where $\sigma_{1}<\sigma_{2}<\cdots<\sigma_{k}$ are the layers of $\sigma$, and let $s_{i}$ be the number of letters in layer $\sigma_{i}$. Let $n=|\sigma|=s_{1}+\cdots+s_{k}$. We get an occurrence of $\pi$ in $\sigma$ by finding an occurrence of $\pi_{1}$ in $\sigma_{i_{1}}, \pi_{2}$ in $\sigma_{i_{2}}, \ldots, \pi_{l}$ in $\sigma_{i_{l}}$ for some $i_{1}<i_{2}<\cdots<i_{l}$; therefore

$$
\begin{aligned}
\nu(\pi, \sigma) & =\sum_{1 \leq i_{1}<\cdots<i_{l} \leq k} \nu\left(\pi_{1}, \sigma_{i_{1}}\right) \cdots \nu\left(\pi_{l}, \sigma_{i_{l}}\right) \\
& \leq \sum_{1 \leq i_{1}<\cdots<i_{l} \leq k} \frac{s_{i_{1}}^{m_{1}}}{m_{1} !} \delta\left(\pi_{1}\right) \cdots \frac{s_{i_{l}}^{m_{l}}}{m_{l} !} \delta\left(\pi_{l}\right) \quad \text { (by Lemma 2) } \\
& =\delta\left(\pi_{1}\right) \cdots \delta\left(\pi_{l}\right) \sum_{1 \leq i_{1}<\cdots<i_{l} \leq k} \frac{s_{i_{1}}^{m_{1}} \cdots s_{i_{l}}^{m_{l}}}{m_{1} ! \cdots m_{l} !} .
\end{aligned}
$$

Now let $\tau_{c}=1^{c s_{1}} \cdots k^{c s_{k}} ;$ then

$$
\begin{aligned}
\sum_{1 \leq i_{1}<\cdots<i_{l} \leq k} \frac{s_{i_{1}}^{m_{1}} \cdots s_{i_{l}}^{m_{l}}}{m_{1} ! \cdots m_{l} !} & =\lim _{c \rightarrow \infty} \sum_{1 \leq i_{1}<\cdots<i_{l} \leq k} \frac{\left(\begin{array}{c}
c s_{i_{1}} \\
m_{1}
\end{array}\right) \cdots\left(\begin{array}{c}
c s_{i_{l}} \\
m_{l}
\end{array}\right)}{c^{m}} \\
& =\lim _{c \rightarrow \infty} \frac{\nu\left(1^{m_{1}} \cdots l^{m_{l}}, \tau_{c}\right)}{c^{m}} \\
& \leq \lim _{c \rightarrow \infty} \frac{\frac{(c n)^{m}}{m !} \delta(\rho)}{c^{m}} \quad \text { (by Lemma 2) } \\
& =\frac{n^{m}}{m !} \delta(\rho) .
\end{aligned}
$$

Combining these results gives

$$
\nu(\pi, \sigma) \leq \delta\left(\pi_{1}\right) \cdots \delta\left(\pi_{l}\right) \frac{n^{m}}{m !} \delta(\rho)=\frac{n^{m}}{m !} \delta_{0},
$$

as claimed. Now $\sigma^{(j)}$ is layered for each $j$ so therefore

$$
\delta(\pi)=\lim _{j \rightarrow \infty} d\left(\pi, \sigma^{(j)}\right)=\lim _{j \rightarrow \infty} \frac{\nu\left(\pi, \sigma^{(j)}\right)}{\left(\begin{array}{c}
\left|\sigma^{(j)}\right| \\
m
\end{array}\right)} \leq \lim _{j \rightarrow \infty} \frac{\frac{\left|\sigma^{(j)}\right|^{m}}{m !}}{\left(\begin{array}{c}
\left|\sigma^{(j)}\right| \\
m
\end{array}\right)} \delta_{0}=\delta_{0},
$$

proving the upper bound.

On the other hand, for certain classes of layered patterns we can explicitly construct families of words which asymptotically attain the bound of Proposition 12, as shown below.

Proposition 13 With the same notation as in Proposition 12, suppose that either the permutation $\left[m_{1}, \ldots, m_{l}\right]$ is simple, or that $m_{i}>1$ for at most one value of $i$. Then

$$
\delta(\pi) \geq \delta(\rho) \delta\left(\pi_{1}\right) \cdots \delta\left(\pi_{l}\right) .
$$

So if $\pi$ also satisfies the conclusion of Conjecture 5 then equality holds. 
Proof. First we consider the case where the permutation $\left[m_{1}, \ldots, m_{l}\right]$ is simple. Let $\varepsilon>0$ and let $N \geq m$. By the definition of a simple permutation, we can find an $n>N$ and $\hat{\tau} \in S_{n}$ with $l$ layers such that

$$
d\left(\left[m_{1}, \ldots, m_{l}\right], \hat{\tau}\right) \geq \delta\left(\left[m_{1}, \ldots, m_{l}\right]\right)-\varepsilon .
$$

By Proposition 8, there is a corresponding nondecreasing word $\tau \in[l]^{n}$ such that $d(\rho, \tau) \geq$ $\delta(\rho)-\varepsilon$. Write $\tau=1^{s_{1}} 2^{s_{2}} \cdots l^{s_{l}}$. For each $j=1, \ldots, l$, let $\sigma_{j} \in[n]^{s_{j}}$ be a word maximizing $\nu\left(\pi_{j}, \sigma_{j}\right)$. By Lemma 2, $\nu\left(\pi_{j}, \sigma_{j}\right) \geq\left(\begin{array}{c}s_{j} \\ m_{j}\end{array}\right) \delta\left(\pi_{j}\right)$. Relabel the symbols of $\sigma_{j}$ so as to use only symbols from the set $\left\{s_{1}+\cdots+s_{j-1}+1, \ldots, s_{1}+\cdots+s_{j}\right\}$; then $\sigma_{1}<\sigma_{2}<\cdots<\sigma_{l}$. Let $\sigma=\sigma_{1} \sigma_{2} \cdots \sigma_{l}$. Then $\sigma \in[n]^{n}$, and the number of occurrences of $\pi$ in $\sigma$ is

$$
\begin{aligned}
\nu(\pi, \sigma) & \geq \nu\left(\pi_{1}, \sigma_{1}\right) \nu\left(\pi_{2}, \sigma_{2}\right) \cdots \nu\left(\pi_{l}, \sigma_{l}\right) \\
& \geq\left(\begin{array}{c}
s_{1} \\
m_{1}
\end{array}\right)\left(\begin{array}{c}
s_{2} \\
m_{2}
\end{array}\right) \cdots\left(\begin{array}{c}
s_{l} \\
m_{l}
\end{array}\right) \delta\left(\pi_{1}\right) \delta\left(\pi_{2}\right) \cdots \delta\left(\pi_{l}\right) \\
& =\nu(\rho, \tau) \delta\left(\pi_{1}\right) \delta\left(\pi_{2}\right) \cdots \delta\left(\pi_{l}\right) .
\end{aligned}
$$

Since $d(\pi, \sigma)=\nu(\pi, \sigma) /\left(\begin{array}{c}n \\ m\end{array}\right)$ and $d(\rho, \tau)=\nu(\rho, \tau) /\left(\begin{array}{c}n \\ m\end{array}\right)$,

$$
\begin{aligned}
d(\pi, \sigma) & \geq d(\rho, \tau) \delta\left(\pi_{1}\right) \delta\left(\pi_{2}\right) \cdots \delta\left(\pi_{l}\right) \\
& \geq(\delta(\rho)-\epsilon) \delta\left(\pi_{1}\right) \delta\left(\pi_{2}\right) \cdots \delta\left(\pi_{l}\right) .
\end{aligned}
$$

So $\delta(\pi, n, n) \geq d(\pi, \sigma) \geq(\delta(\rho)-\epsilon) \delta\left(\pi_{1}\right) \delta\left(\pi_{2}\right) \cdots \delta\left(\pi_{l}\right)$. Since this holds for all $n>$ $N$, we obtain $\delta(\pi) \geq(\delta(\rho)-\epsilon) \delta\left(\pi_{1}\right) \delta\left(\pi_{2}\right) \cdots \delta\left(\pi_{l}\right)$, and $\epsilon>0$ is arbitrary so $\delta(\pi) \geq$ $\delta(\rho) \delta\left(\pi_{1}\right) \delta\left(\pi_{2}\right) \cdots \delta\left(\pi_{l}\right)$ as claimed.

The case where $m_{i}>1$ for at most one value of $i$ is similar. Say $m_{i}=1$ for $i \neq i_{0}$. Let $n \geq m$ and let $\tau \in[n]^{n}$ be a $\rho$-maximizer; since $\rho$ is nondecreasing we may take $\tau$ to be nondecreasing. By Lemma $2, \nu(\rho, \tau) \geq\left(\begin{array}{c}n \\ m\end{array}\right) \delta(\rho)$. Write $\tau=1^{s_{1}} 2^{s_{2}} \cdots r^{s_{r}}$. For each $j=1$, $\ldots, r$, let $\sigma_{j} \in[n]^{s_{j}}$ be a word maximizing $\nu\left(\pi_{i_{0}}, \sigma_{j}\right)$. By Lemma $2, \nu\left(\pi_{i_{0}}, \sigma_{j}\right) \geq\left(\begin{array}{c}s_{j} \\ m_{j}\end{array}\right) \delta\left(\pi_{i_{0}}\right)$, and for $i \neq i_{0}, m_{i}=1$ so $\nu\left(\pi_{i}, \sigma_{j}\right)=s_{j}=\left(\begin{array}{c}s_{j} \\ m_{i}\end{array}\right)$. Relabel the symbols of $\sigma_{j}$ so as to use only symbols from the set $\left\{s_{1}+\cdots+s_{j-1}+1, \ldots, s_{1}+\cdots+s_{j}\right\}$; then $\sigma_{1}<\sigma_{2}<\cdots<\sigma_{l}$. Let $\sigma=\sigma_{1} \sigma_{2} \cdots \sigma_{l}$. Then $\sigma \in[n]^{n}$, and

$$
\begin{aligned}
\nu(\pi, \sigma) & =\sum_{1 \leq j_{1}<\cdots<j_{l} \leq r} \nu\left(\pi_{1}, \sigma_{j_{1}}\right) \cdots \nu\left(\pi_{l}, \sigma_{j_{l}}\right) \\
& \geq \sum_{1 \leq j_{1}<\cdots<j_{l} \leq r}\left(\begin{array}{c}
\sigma_{j_{1}} \\
m_{1}
\end{array}\right) \cdots\left(\begin{array}{c}
\sigma_{j_{l}} \\
m_{l}
\end{array}\right) \delta\left(\pi_{i_{0}}\right) \\
& =\nu(\rho, \tau) \delta\left(\pi_{i_{0}}\right) \\
& =\nu(\rho, \tau) \delta\left(\pi_{1}\right) \cdots \delta\left(\pi_{l}\right)
\end{aligned}
$$

since $\delta\left(\pi_{i}\right)=1$ for $i \neq i_{0}$. It follows that

$$
\delta(\pi, n, n) \geq d(\pi, \sigma) \geq d(\rho, \tau) \delta\left(\pi_{1}\right) \cdots \delta\left(\pi_{l}\right) \geq \delta(\rho) \delta\left(\pi_{1}\right) \cdots \delta\left(\pi_{l}\right) .
$$

Since $n$ is arbitrary, $\delta(\pi) \geq \delta(\rho) \delta\left(\pi_{1}\right) \cdots \delta\left(\pi_{l}\right)$. 
Corollary $14 \delta\left(1^{p} 3^{q} 2^{r}\right)=\delta([p, q+r]) \delta([q, r])$.

Proof. Write $\pi=1^{p} 3^{q} 2^{r}=\pi_{1} \pi_{2}$ where $\pi_{1}=1^{p}, \pi_{2}=3^{q} 2^{r}$ and $\rho=1^{p} 2^{q+r}$. By Theorem 9 , the condition of Proposition 12 holds. When $p>1$, the permutation $[p, q+r]$ is simple by Theorem 11, and when $p=1$, the permutation $[p, q+r]$ has only one part of size greater than one; hence the condition of Proposition 13 holds as well. So we conclude that

$$
\delta(\pi)=\delta(\rho) \delta\left(\pi_{1}\right) \delta\left(\pi_{2}\right)=\delta\left(1^{p} 2^{q+r}\right) \delta\left(1^{p}\right) \delta\left(3^{q} 2^{r}\right)=\delta([p, q+r]) \delta([q, r]),
$$

as claimed.

Using the formulas $\delta([r, s])=\left(\begin{array}{c}r+s \\ r\end{array}\right) \frac{r^{r} s^{s}}{(r+s)^{r+s}}$ for $r, s \geq 2$ and

$$
\delta([r, 1])=r(1-\alpha) \alpha^{r-1}, \quad \text { where } 0<\alpha<1,(1-r \alpha)^{r+1}=1-(r+1) \alpha,
$$

we can compute exactly the packing density of any pattern of the form $1^{p} 3^{q} 2^{r}$.

Corollary 15 Let $\bar{\pi}=\left[a_{1}, \ldots, a_{l}\right]$ be a layered permutation, and let $\pi$ be the permutation obtained by repeating each letter of $\bar{\pi} r$ times. If $\left[r a_{1}, \ldots, r a_{l}\right]$ is simple, then $\delta(\pi)=$ $\delta\left(\left[r a_{1}, \ldots, r a_{l}\right]\right) \delta\left(\pi_{1}\right) \cdots \delta\left(\pi_{l}\right)$, where $\pi_{i}$ is the layered permutation with $a_{i}$ layers of size $r$.

Proof. Follows from Theorem 7 and Propositions 12 and 13.

\section{Acknowledgements}

This research was done at the University of Minnesota Duluth Research Experience for Undergraduates with the support of NSF grant DMS-0137611 and NSA grant MDA90402-1-0060. A special thanks is due to Joseph A. Gallian, the director of the Duluth REU. I would also like to thank Geir Helleloid and Phil Matchett for many helpful comments.

\section{References}

[1] M. H. Albert, M. D. Atkinson, C. C. Handley, D. A. Holton, W. Stromquist, On packing densities of permutations, Electron. J. Combin. 9 (2002), \#R5.

[2] A. Burstein, P. Hästö, T. Mansour, Packing patterns into words, Electron. J. Combin. 9(2) (2003), \#R20.

[3] Peter A. Hästö, The packing density of other layered patterns, Electron. J. Combin. 9(2) (2003), \#R1. 